\title{
RESEARCH ON THE DIGITAL RETENTION MECHANISM OF TIBETAN BUDDHISM ARCHITECTURE BASED ON UAV AND TLS: A CASE STUDY OF BAOGUANG HALL
}

\author{
Zongfei $\mathrm{Li}^{1,3}$, Miaole $\mathrm{Hou}^{2,3} *$, Youqiang Dong ${ }^{2,3}$, Jian Wang ${ }^{2,3}$, Yuhang $\mathrm{Ji}^{2,3}$, Pengpeng Huo ${ }^{2,3}$ \\ 1 Architecture Department, Beijing University of Civil Engineering and Architecture, No.1, Zhanlan Road, Xicheng District, \\ Beijing 100044, China; lzfbucea@163.com \\ 2 School of Geomatics and Urban Spatial Informatics, Beijing University of Civil Engineering and Architecture, Beijing 100044, \\ China; wangjian@bucea.edu.cn(J.W.); 2108521519033@stu.bucea.edu.cn (Y.J.); 2208521518001@stu.bucea.edu.cn (P.H.) \\ 3 Beijing Key Laboratory For Architectural Heritage Fine Reconstruction \& Health Monitoring, No.15Yongyuan Road, Daxing \\ District, Beijing, 102616 \\ * Correspondence: houmiaole@bucea.edu.cn
}

KEY WORDS: UAV and TLS, Tibetan Buddhist architecture; Point cloud; Multi-dimensional retention

\begin{abstract}
:
Tibetan Buddhist architecture embodies ancient Chinese architectural culture and religious culture. In the past, the information retention mechanisms for ancient buildings were implemented as photos, tracings, and rubbings, which cannot fundamentally document the authenticity of architectural heritage. To explore the digital retention method for the unique style of Han Tibetan architecture, this research that based on the idea of reverse documentation first collects point cloud data with the technical support of unmanned aerial vehicle(UAV) photogrammetry and terrestrial laser scanner(TLS) and then uses registration method to obtain the integral of the point cloud model of Baoguang Hall. This paper explores the possibility of extracting 2D and 3D information, such as architectural plans, facades, decorative components, and models of the temple architecture, by processing point cloud data. Finally, this study proves the feasibility of using digital technology for the preservation and protection of architectural heritage.
\end{abstract}

\section{INTRODUCTION}

The Ganqing area is located in the northwest of China. Due to many multi-ethnic people living here and the presence of several religions (Deng,2017), a unique and diversified architectural culture was formed in the area. Unlike the architectural styles of other regions, the Ganqing area formed a distinct architectural expression of the Han-style under the influence of Buddhist culture (Muya,1999). Baoguang hall is a typical work of Han-style Tibetan Buddhist architecture in Qutan Temple, Ledu County, Qinghai Province, China (Song,2014).

At present, architectural research is mainly focused on decorative murals and Tibetan architectural culture (Cui,2014). However, there are relatively few studies on the construction principles of Tibetan Buddhist architecture in the Han style. With the development of digital technology, traditional optical measurement instruments have been replaced with modern surveying and mapping methods integrating light, machines, electricity, and calculations (Athey,2008). With the emergence of UAV photogrammetry and three-dimensional (3D) laser scanners, digital equipment and technology have been widely applied to the $3 \mathrm{D}$ modeling of cultural relics and data collection from ancient sites.

For example, in the field of ancient site protection, Chen et al. proposed a measurement method for the Ming Great Wall using the technologies of field surveys and UAV photogrammetry as early as 2008 (Eisenbeiss,2006). The key application of this method was to investigate the attributes and distribution of the Great Wall via field survey and to measure the length of the Great Wall via UAV photogrammetry. In the field of cultural relic protection, Scopigno et al. successfully reconstructed a $3 \mathrm{D}$ model for the statue of David by using semi-automatic 3D scanning technology [9]. Hou et al. solved the absence of evidence for restoring the Dazu Thousand-Hand Bodhisattva finger by proposing a virtual restoration method based on multiscale spatial geometric features (Hou,2018).

In addition to the many efforts that researchers have made in the field of cultural relics and sites, in recent years, researchers have explored the methods of UAV photogrammetry and 3D laser scanning for protecting ancient architecture. These results of surveying and mapping were initially applied to three different aspects: data collection, digital restoration, and the historical value of the architectural heritage (Lee,2018). The protection of ancient architectural heritage involves the integration of multiple disciplines, especially for the interpretation of ancient architecture's ontology, which includes technical support from the disciplines of surveying and mapping alongside support from elements of the architecture field, such as architectural history, culture, and construction rules:

In terms of data collection, the emergence of low-cost UAV and structural self-motion (SfM) algorithms has provided great convenience for data acquisition. Sun et al. used this method to overcome the difficulties of data collection in the high-altitude areas of Tibet and completed overall mapping of the outer surface of the Multi-Door Stupa and the surrounding environment (Sun,2018). In addition, the portable and flexible characteristics of TLSs can be used as a supplement to obtain dense and accurate geometric information inside a building, which can be easily integrated with high-resolution data (Remondino,2011).

In terms of digital restoration, a combination of TLS and UAV photogrammetry technology can help restore complex architectural heritage. $\mathrm{Xu}$ et al. successfully integrated two

\footnotetext{
* Corresponding author: houmiaole@bucea.edu.cn
} 
types of 3D point clouds captured by UAV and TLS through multivariate data fusion technology, thereby realizing the overall restoration of a historic building in Fujian Province, China (Xu,2014). Chen took an ancient watchtower in Tibet Province, China as an example. Chen used the data fusion technology of UAV and a 3D laser scanner to create a damaged state model and fully explored the form and construction information of the surrounding watchtowers. On this basis, Chen restored the damaged state model of the watchtower to its original appearance by analyzing the information of its form and craftsmanship. The digital restoration result perfectly interprets the military defense and mysterious religious sacrificial functions of the ancient watchtower (Chen,2018). For a disappeared ancient plank road site, Chen et al. proposed a construction mechanics analysis method based on the current accurate $3 \mathrm{D}$ data of the plank road and successfully deduced the size of each component and the restored model (Chen,2016).

In terms of historical value interpretation, digitization is an important medium for the dissemination of architectural historical value and educational guidance (Arayici,2007). Gabellone et al. developed an immersive 3D site platform based on point cloud data, which enables visitors to interact with $3 \mathrm{D}$ models in real time and intuitively experience the historical connotations of architectural sites (Gabellone,2013). Enrica and Alessandro successfully realized a virtual display for the incomplete facade of the church of San Lorenzo in Montedinove through a video projection to restore and amplify the cultural, historical, and symbolic value of the ancient architecture (Petrucci,2017).

Although many of the above studies have made efforts at different levels to study architectural heritage, they have not applied digital analyses or related research to the multidimensional retention mechanism. Based on this factor, we specifically selected the Tibetan Buddhist architecture in the Ganqing area of China as the case of this study.

Tibetan Buddhist buildings are mainly composed of wooden structures in the Ganqing area, forming a wooden frame structure characterized by wooden columns, beams, windows, doors, and a roof. However, as time passes, as important elements of cultural heritage, wooden buildings are facing major challenges in their protection, restoration, and maintenance (Chun,2015). As an ancient wooden building with important historical, cultural, and religious value in Tibetan Buddhism, Baoguang hall needs to be protected and properly valued by the public.

The general objective of this paper is to propose, using digitization, a multi-dimensional information retention mechanism for ancient wooden buildings of Tibetan Buddhism in the Han style. In the past, the information retention mechanisms for ancient buildings were implemented as photos, tracings, and rubbings, which cannot fundamentally document the authenticity of architectural heritage. Based on the idea of reverse documentation, this paper explores the possibility of extracting 2D and 3D information, such as architectural plans, facades, decorative components, and models of the temple architecture, by processing point cloud data. Moreover, this research seeks to analyze the construction principles of temple architecture and the influence of Tibetan Buddhist culture on decorative components from an architectural perspective.

To reach this objective, a practical case study was designed in which the following specific sections were set:

First, we defined the research object and described in detail the location and historical evolution of the Baoguang hall. At the same time, the latest pictures also revealed the hall's current situation.

Under the background of the preliminary investigation, the next task of data acquisition includeed using the UAV and TLS to respectively obtain the external and internal point cloud data of Baoguang hall and then fuse these point cloud data.

Based on the previous section, the fused point cloud data were used to create a digital fine 3D model of the Baoguang hall, and the point cloud projection method was used to obtain the architectural plan, façade, and section information.

Finally, in the research results section, architectural knowledge was used to interpret the construction principles of Baoguang hall, promoting people's understanding of Tibetan Buddhist architecture in the Han style. The beneficial effects and value of the digital multi-dimensional retention mechanism are discussed at the end of the article.

\section{RESEARCH OBJECT}

\subsection{Baoguang Hall}

Baoguang hall is a classical Han-style Tibetan Buddhist building in the Ganqing area. It is located in the middle of Qutan Temple, Ledu County, Qinghai Province, China (Figure 1), and was built in the Yongle year (1418) of the Ming Dynasty (1368 - 1644 AD) (Sezo,1979). The Qutan Temple was constructed because emperor Zhu $\mathrm{Di}$ in the Ming Dynasty wanted to consolidate the religious predominance of Qutan Temple. Hence, the emperor ordered the eunuch and commander to be responsible for the construction of Qutan Temple. In the early Ming Dynasty, this temple played an important role in promoting frontier governance and national unity. Today, it has become one of the most well-preserved examples of Ming dynasty architecture in Northwest China and was counted among the second batch of national key cultural relic protection units by the State Council in 1982 (Zhang,1964).

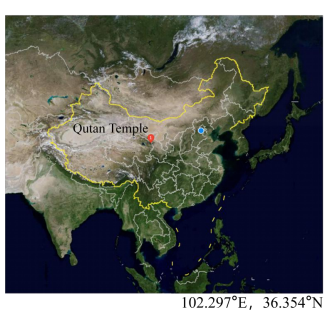

(a)

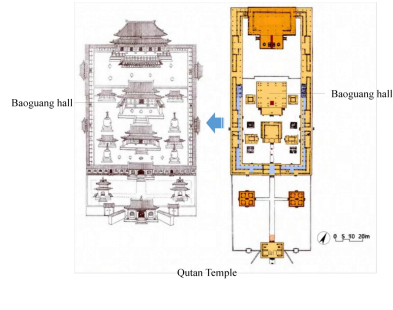

(b)
Figure 1. (a) Qutan Temple is located in Ledu County, Qinghai Province; (b) the location of Baoguang hall in Qutan Temple(Sun,2018).

\subsection{Threats}

As a Han style building in a Tibetan Buddhist temple, the Baoguang hall is facing significant challenges in its protection. In terms of nature, Ledu county is located in a continental semiarid climate area, and the climatic features of drought and solar radiation in the area can easily cause cracks and deformations of wooden building components. In terms of protection, Baoguang hall faces the irreversible corrosion of its eaves caused by bird droppings. Therefore, it is necessary to carry out fine surveying and mapping to establish a multi-dimensional retention mechanism and thereby prevent the gradual loss of information on ancient buildings. 


\section{RESEARCH STRATEGY}

\subsection{Technical Process}

The technical process of this research was to use UAV photogrammetry to obtain external data for Baoguang hall. In addition, UAV photogrammetry was combined with TLS to obtain the internal data of Baoguang hall. Then, the two types of point cloud data were fused into a set of cloud data. Next, the point cloud data were further processed in two steps. One used the point cloud data to construct a triangular mesh and obtain a real 3D model of Baoguang hall through texture mapping (Hu,2016); the second involved importing point cloud data into CAD2014 and using point cloud projection and slicing functions to obtain architectural plan drawings, facade drawings, and section line drawings of Baoguang hall. The workflow and process of data collection are shown in Figure 2.

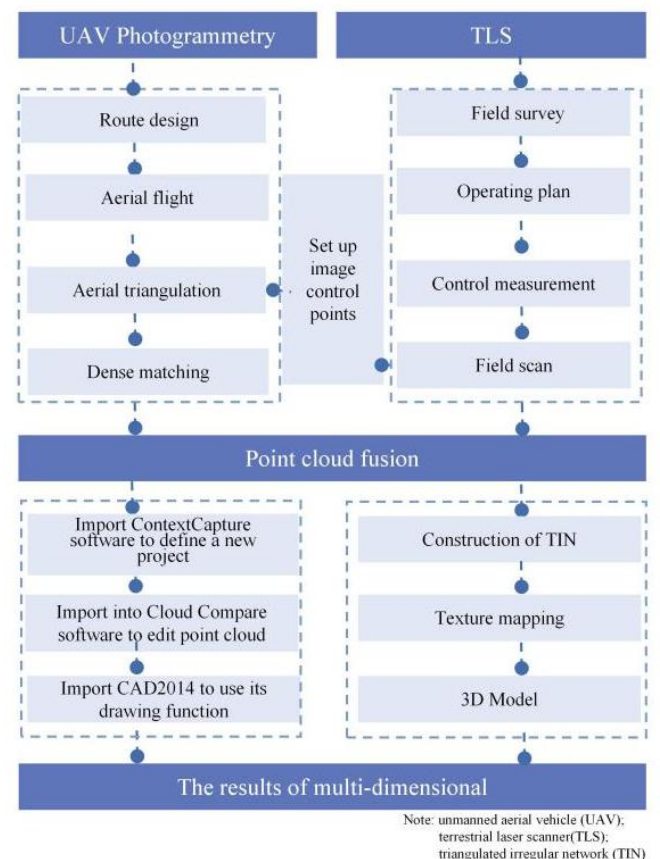

Figure 2. The workflow and process of data collection.

\subsection{Data Collection}

\subsubsection{Data Collection from the External area of Baoguang Hall by using UAV}

At the scene, a professional 4-axis aircraft of DJI Phantom 4 Pro (DJ-Innovations Inc., Shenzhen, China) was used for surveying and mapping Baoguang hall. This UAV's maximum flying altitude can reach $6000 \mathrm{~m}$, which meets the flight altitude requirements of Qutan Temple at a $2558 \mathrm{~m}$ sea level. The camera model of the UAV is FC6310, as shown in Table 1. To better achieve the required quality of data collection, the camera's parameters should meet the following standards: (1) The ISO value should be adjusted as low as possible to avoid overexposure of the photo due to a high ISO; (2) a small aperture camera should be employed to obtain clear photos with depth of field; (3) the shutter speed should not be too slow, otherwise the photos will appear blurred due to movement.

\begin{tabular}{|l|l|}
\hline The Camera Parameters & Value \\
\hline Manufacturer & DJI \\
Type & FC6310 \\
Aperture value & F/5.6 \\
Exposure time & $1 / 500 \mathrm{~s}$ \\
ISO speed & ISO-100 \\
Focal length & $8.8 \mathrm{~mm}$ \\
Maximum aperture & 2.97 \\
Sensor size & $13.2 \mathrm{~mm}$ \\
\hline
\end{tabular}

Table 1. Parameters of the camera carried by the unmanned aerial vehicle (UAV).

After 5 flight tests, the aerial route of layered shooting was finally selected, as shown in Figure 3. The flight path was divided into four layers, lower, middle, upper, and top, and the entire flight duration was 6 min. Initially, the UAV followed a pre-set aerial route and obtained a total of 231 pictures. However, to meet the demands of 3D model reconstruction, we removed pictures with an overlap rate of less than $66 \%$ and ultimately retained 225 usable pictures, whose image information is shown in Table 2.

\begin{tabular}{|l|c|c|}
\hline Photo & Photo ID & Value \\
\hline & $\begin{array}{c}\text { resolution } \\
\text { width } \\
\text { height } \\
\text { horizontal } \\
\text { resolution } \\
\text { vertical } \\
\text { resolution } \\
\text { color }\end{array}$ & 76472 pixel \\
& 7648 pixel \\
& sRGB \\
\hline
\end{tabular}

Table 2. Parameters of the camera carried by the UAV.

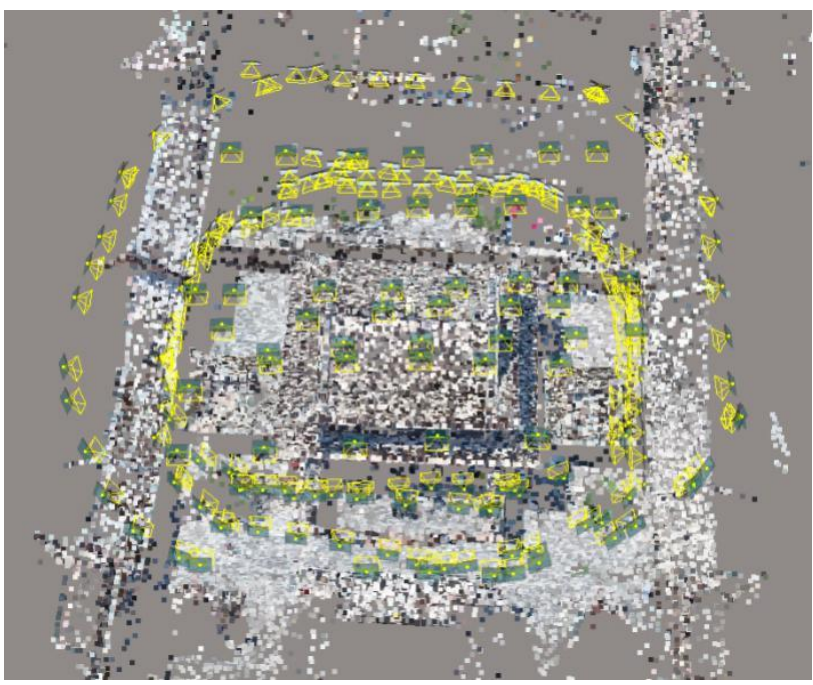

Figure 3. The aerial route of the UAV collecting image data.

The UAV has a position and positioning system (POS), allowing it to be positioned in the WGS-84 coordinate system. 
ContextCapture software automatically processes 225 images over about $7.3 \mathrm{~h}$ to generate point cloud data. The software has a camera calibration function, and the Initial Values represent the original internal orientation element of the camera, and Optimized Values are the corrected internal orientation. According to the specifications, the margin of error is less than $1 \mathrm{~mm}$, which meets the application requirements.

\subsubsection{Data Collection of the Ancient Structure and Decorative Components by TLS}

It is most convenient to collect data on the internal building structure and partial decorative components of Baoguang hall by using TLS. First, TLS was used to scan the indoor beamcolumn structure and the decorative components of the building facade. Secondly, the automatic filtering software (Cyclone V7.0, Leica Geosystems AG, Heerbrugg, Switzerland) was used to filter the point cloud data to eliminate noise and remove useless information.

\subsubsection{Data Fusion}

Next, we needed to define and submit a new production project in the ContextCapture software and output the project model to the LAS point cloud format to ensure the exchange of point cloud data between different platforms. This process ensured the correct fusion of the two types of point clouds. UAV photogrammetry point cloud data and 3D laser scanning point cloud data were converted to the universal *. las format. Since the accuracy of the 3D laser point cloud is much higher than that of oblique photogrammetry, in the registration and fusion of the two point cloud data, the 3D laser point cloud was employed as the benchmark, and the ICP algorithm and manual registration method were used to register the two point cloud data to obtain a high-precision fusion point cloud model.

\section{THE RESULT OF DATA PROCESSING}

\subsection{Retention of the 3D Architectural Model}

After the aerial triangulation is calculated, and the images are densely matched, the point clouds of all images can be calculated to generate a triangulated irregular network (TIN), which then forms a blank model. To ensure the output effect of the model, the point cloud data cannot be compressed. The software extracts the corresponding texture from the image and automatically maps the texture to the white model. After the project was successfully submitted, a 3D point cloud model in *. las format was generated, as shown in Figure 4.

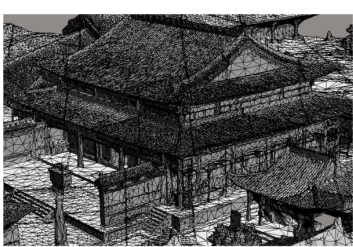

(a)

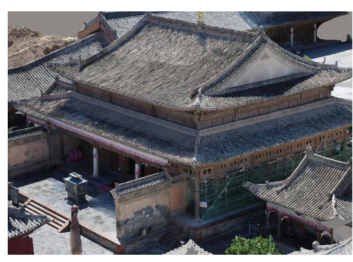

(c)

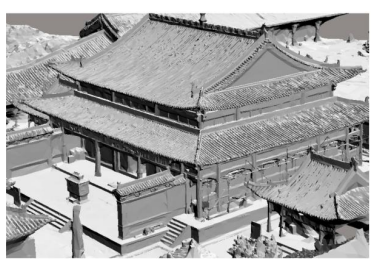

(b)

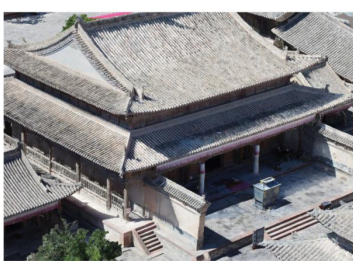

(d)
Figure 4. Point cloud data processing of Baoguang hall: (a) Irregular triangulation generated by the point cloud; (b) blank model; (c) texture mapping to the 3D model; (d) 3D model.

\subsection{Retention Information of the Architectural Plan and Facade}

This study proposes an extraction method for 2D architecture information based on point cloud projection. In this research, we imported the 3D point cloud model into the Cloud Compare (a 3D point cloud processing software) software and used the powerful editing functions of this software to remove obstructions around Baoguang hall. This editing function can reduce a large amount of useless point cloud data through cropping. Then, we saved the point cloud model in the *.pcg format.

The user imports the point cloud data in the *.pcg format into CAD2014, as shown in Figure 5. In the 3D space model, the views of the model were adjusted to the front view and top view (Figure 6a1,b1). The point cloud density was set to the maximum, and the color of the point cloud mapping was set to grayscale mode. Then, the $2 \mathrm{D}$ facade and floor plan of the Baoguangdian hall were extracted according to the sparse value of the point cloud under the layout function of CAD2014, as shown in Figure 6a2,b2.

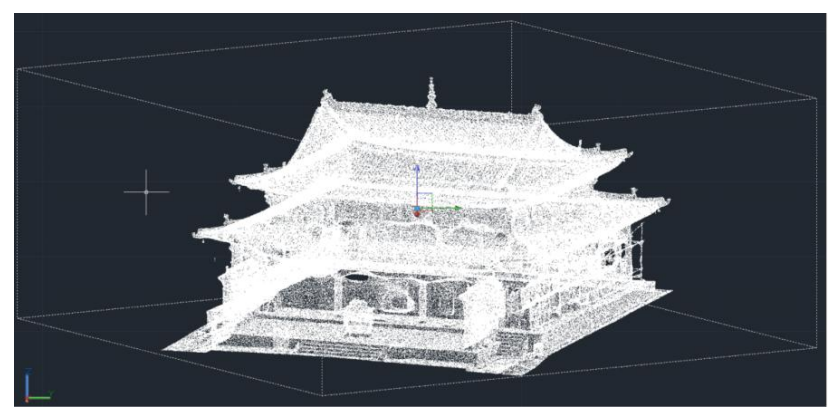

Figure 5. The point cloud data of Baoguang hall in the CAD2014.

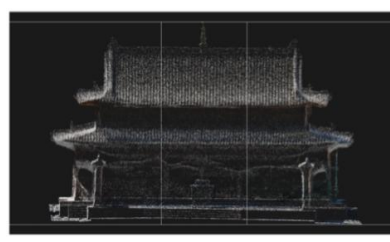

(a1)

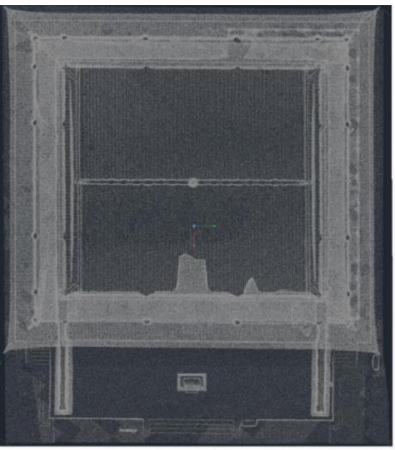

(b1)

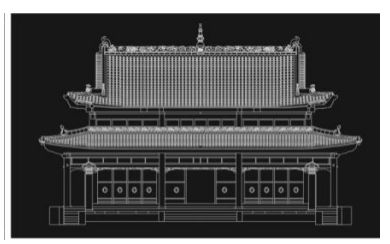

(a2)

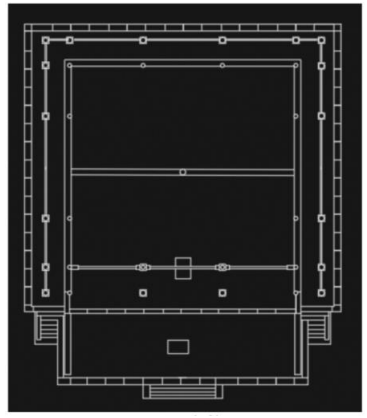

(b2)
Figure 6. The extraction results of the point cloud information: (a1) Facade of the point cloud of Baoguang hall; (a2) extracted facade line drawing from the point cloud; (b1) plan of the point 
cloud of Baoguang hall; (b2) extracted plan line drawing from the point cloud.

\subsection{Retention of a Building Cross-Section Drawing}

The complete Baoguang hall 3D model was reconstructed based on the fusion data of existing UAV point cloud data and TLS point cloud data. According to the idea of reverse documentation, in CAD2014, the point cloud cutting function was used to cut the $3 \mathrm{D}$ model at a position that could reflect the architectural structure, as shown in Figure 7a. Then, we used the point cloud projection function to map the Baoguang hall section to the layout function and manually extracted the Baoguang hall architectural section view (Figure 7b).

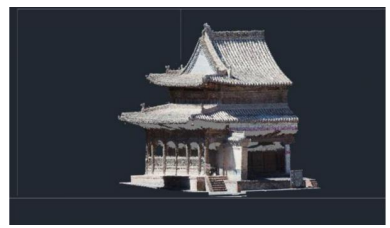

(a)

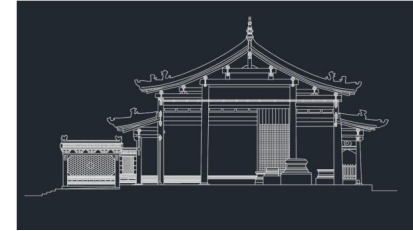

(b)
Figure 7. (a) Cutting the point cloud of Baoguang hall along its structural frame; (b) extracting the cross-section of Baoguang hall through point cloud mapping.

\subsection{Retention of Architectural Decorative Components}

Based on the flexible, mobile, and high-precision features of the TLS, the decorative components of Baoguang hall were individually and finely scanned. Based on the extraction thinking of reverse documentation, we gradually extracted the point cloud data of the decorative components on the roof of Baoguang hall, such as Baoping (宝瓶), Chiwen (鸱吻), Chuishou (垂兽), Qiangshou (戗兽), and brick sculptures (砖 雕), as shown in Figure 8.

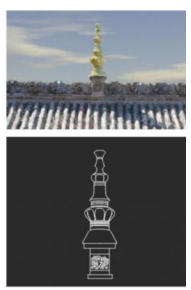

(a)

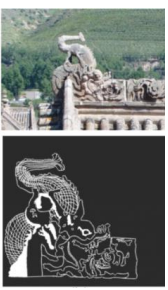

(b)

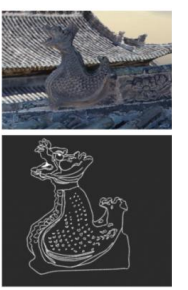

(c)

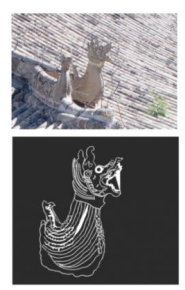

(d)
Figure 8. (a) Retention information of the decorative components: (a) Baoping; (b) Chiwen; (c) Chuishou; (d) Qiangshou.

\section{DISCUSSION}

We abandoned traditional surveying and mapping methods and adopted the integrated application method of drones and ground laser scanners, which provided the multi-dimensional retention advantages of importing 3D point cloud data into CAD software to preserve architectural heritage.

Point cloud data can reflect the authenticity of ancient buildings. We actively explored the support function of AutoCAD2014 for point cloud data and realized the new function of transforming a $3 \mathrm{D}$ view into a $2 \mathrm{D}$ view in AutoCAD2014. For example, the point cloud density can be set to its maximum in the point cloud menu, and the point cloud intensity can be set to grayscale mode In the layout function module, we successfully extracted the elevation of the building by setting the sparse value of the point cloud. In addition, point cloud projection was used to map a section of the ancient building to the layout function of AutoCAD2014, and then we manually extracted the section structure information of the ancient building.

\section{DISCUSSION}

This research did not employ the traditional protection methods of manual surveying and mapping/drawing archiving and instead proposed a new mechanism of authenticity retention based on heritage digitization technology. This method can make full use of modern surveying and mapping methods and combine these methods with reverse modeling thinking to expand the possibilities for digital authenticity retention. The conclusions are mainly reflected in the following three aspects. Han-style Tibetan Buddhist architecture is characterized by complex structures and exquisite forms. For the protection of Han-style Tibetan Buddhist architecture, in terms of technology, quality, labor costs, and time costs, traditional surveying and mapping methods have no advantages compared to new, more technical, methods. The integrated application method using both UAV and TLS can perfectly solve the problem of fine measurements for complex ancient buildings.

Point cloud data have the remarkable characteristics of preserving authentic information on architectural heritage. After the point cloud characteristics are combined with the CAD2014 drawing characteristics, the produced $2 \mathrm{D}$ drawings can not only meet the traditional needs of the cultural protection department for retention but can also more scientifically record the structural characteristics of architectural heritage. In addition, the 3D model is a twin result of the digitization of architectural heritage. Digitization not only provides a data management function for architectural heritage but also represents the best way to perfectly record the current state of a type of architectural heritage.

\section{ACKNOWLEDGEMENTS}

This work is supported by Youth Beijing Scholar Program, Scientific Research Program Project of Beijing Education Commission (KM202110016005) and Natural Science Foundation of Beijing Province (KZ202110016021).

\section{REFERENCES}

Deng, X.; Cao, L.; Li, J. Research on characteristics of Xinjiang traditional architecture in Qing Dyasty: Set of carved boards and curved hiprafter. J. Shenyang Jianzhu Univ. (Soc. Sci.), 2017, 02, 150-153.

Muya, Q.J. Historical Development, Species Analysis and Structural Characteristics of Tibetan Architecture. Collect. Essays Archit. Hist. 1999, 11, 15-25.

Song, Z.R. The Conservation Zone and Constructive Regulation Research of Qutan Temple; Xi ' an University of Architecture and Technology: Xi'an, China, 2014.

Cui, W.H.; Yu.Y. Multiple Symbiosis: A Study on the Cultural Diversity of Qinghai's Vernacular House Architecture. South. Archit. 2014, 6, 60-65.

Athey, J.E.; Freeman, L.K.; Woods, K.A. The transition from traditional to digital mapping: Maintaining data quality while increasing geologic mapping efficiency in Alaska. Transition $2008,11,2$ 
Eisenbeiss, H.; Zhang, L. Comparison of DSMs generated from mini UAV imagery and terrestrial laser scanner in a cultural heritage application. International Archives of the Photogrammetry. Remote Sens. Spat. Inf. Sci. 2006, 36, 90-96. Hou, M.; Yang, S.; Hu, Y.; Wu, Y.; Jiang, L.; Zhao, S.; Wei, P. Novel method for virtual restoration of cultural relics with complex geometric structure based on multiscale spatial geometry. ISPRS Int. J. Geo-Inf. 2018, 7, 353

Lee, J.S.; Lee, I. The 3D Modeling Data Production Method Using Drones Photographic Scanning Technology. J. Korea Inst. Inf. Commun. Eng. 2018, 22, 874-880.

Sun, Z.; Zhang, Y.Y. Using drones and 3D modeling to survey Tibetan architectural heritage: A case study with the multi-door stupa. Sustainability 2018, 10, 2259.

Remondino, F. Heritage recording and 3D modeling with photogrammetry and 3D scanning. Remote Sens. 2011, 3, $1104-1138$

Xu, Z.; Wu, L.; Shen, Y.; Li, F.; Wang, Q.; Wang, R. Tridimensional reconstruction applied to cultural heritage with the use of camera-equipped UAV and terrestrial laser scanner. Remote Sens. 2014, 6, 10413-10434.

Chen, S., Yang, H., Wang, S., Hu, Q. Surveying and Digital Restoration of Towering Architectural Heritage in Harsh Environments: A Case Study of the Millennium Ancient Watchtower in Tibet. Sustainability. 2018, 10, 3138.

Chen, S.; Hu, Q.; Wang, S.; Yang, H. A virtual restoration approach for ancient plank road using mechanical analysis with precision 3D data of heritage site. Remote Sens. 2016, 8, 828.

Arayici, Y. An approach for real world data modelling with the 3D terrestrial laser scanner for built environment. Autom. Constr. 2007, 16, 816-829.

Gabellone, F.; Ferrari, I.; Giannotta, M. T.; Dell'Aglio, A. From museum to original site: A $3 \mathrm{~d}$ environment for virtual visits to finds re-contextualized in their original setting. In: 2013 Digital Heritage International Congress (DigitalHeritage). 2013, 2, 215 222 .

Petrucci, E.; Vannicola, A. Incompleta Reficere: The Incomplete Façade of the Church of San Lorenzo in Montedinove and Its Virtual Image. In Multidisciplinary Digital Publishing Institute Proceedings. 2017, 1, 881.

Chun, Q.; Van Balen, K.; Pan, J.; Sun, L. Structural performance and repair methodology of the Wenxing lounge bridge in China. International Journal of Architectural Heritage. 2015, 9, 730-743.

Sezo. Qinghai Ledu Temple Collo. J. Qinghai Inst. Natl. 1979, Z1, 20-33.

Zhang, Y.X.; Du, X.Z. Qinghai Ledu Temple Survey Report. Cult. Relics 1964, 5, 46-60.

Sun, X.X.; Chen, W. The Cross-Regional Inheritance of Craftmanship in Early Ming Dynasty: A Study on Masonry in Qutan Temple in Comparison with Nanjing and Beijing. Architectural Journal. 2018, 2, 22-27.

Hu, Q.; Wang, S.; Fu, C.; Ai, M.; Yu, D.; Wang, W. Fine surveying and $3 \mathrm{D}$ modeling approach for wooden ancient architecture via multiple laser scanner integration. Remote Sens. 2016, 8, 270 . 